\title{
SU(2) gauge theory with many flavors of domain-wall fermions
}

\author{
Hideo Matsufuru* \\ High Energy Accelerator Research Organization (KEK), Tsukuba 305-0801, Japan, \\ and Graduate University for Advanced Studies (Sokendai), Tsukuba 305-0801, Japan \\ E-mail: hideo.matsufuruekek.jp
}

\section{Kei-ichi Nagai}

Kobayashi-Maskawa Institute for the Origin of Particles and the Universe (KMI), Nagoya

University, Nagoya, 464-8602, Japan

E-mail: keiichi.nagaiekmi.nagoya-u.ac.jp

\section{Norikazu Yamada}

High Energy Accelerator Research Organization (KEK), Tsukuba 305-0801, Japan, and Graduate University for Advanced Studies (Sokendai), Tsukuba 305-0801, Japan E-mail: norikazu.vamadadkek.jp

We study the SU(2) gauge theory with many flavors of fermions in the fundamental representation. With the Iwasaki gauge action and the standard domain-wall fermion action, dynamical simulations with $N_{f}=2,4,6,8$ are being performed, at least, at two values of $\beta$ and three values of fermion mass for each $N_{f}$. The preliminary results of the static potential, the residual mass of the domain-wall fermions and meson spectrum are presented with an emphasis on the $N_{f}$ dependence.

The 32nd International Symposium on Lattice Field Theory

23-28 June, 2014

Columbia University New York, NY

\footnotetext{
*Speaker.
} 


\section{Introduction}

The SU(2) gauge theory has recently been drawn revised interest in search for a theory beyond the standard model (SM). As a candidate of walking technicolor [U, ㅁ], which may resolve not only conceptual drawbacks including the fine-tuning problem but also practical deficit such as absence of dark matters in the SM, its phase structure with respect to the number of flavors has been nonperturbatively studied in lattice simulations [3]

In a viewpoint of chiral symmetry breaking, the $\mathrm{SU}(2)$ gauge theory is unique as the symmetry breaking pattern is different from QCD. Because of the pseudo-reality of of the $\mathrm{SU}(2)$ group, for fermions in the fundamental representation, the breaking pattern is $S U\left(2 N_{f}\right) \rightarrow S p\left(2 N_{f}\right)$, while for $\mathrm{SU}(\mathrm{N})$ with $N \geq 3$ it is $S U\left(N_{f}\right) \times S U\left(N_{f}\right) \rightarrow S U\left(N_{f}\right)$.

The SU(2) gauge theory with fundamental fermions has been already studied on the lattice

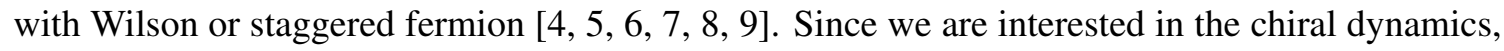
it is natural to choose a fermion action which retains the chiral symmetry on the lattice as much as possible. Although the overlap fermion operator holds an exact chiral symmetry, its numerical cost and complexity in setup are beyond the computational capability available to us. We adopt in this work the domain-wall fermion action that keeps numerical cost modest while its chiral symmetry breaking is under good control.

The domain-wall fermion action is written as

$$
\begin{gathered}
S_{D W}=\sum_{x, s, y, s^{\prime}} \bar{\psi}(x, s) D_{D W}\left(x, s ; y, s^{\prime}\right) \psi\left(y, s^{\prime}\right), \\
D_{D W}\left(x, s ; y, s^{\prime}\right)= \\
D_{W}\left(x, y ;-M_{0}\right) \delta_{s, s^{\prime}} \\
-\frac{1}{2}\left[\left(1-\gamma_{5}\right) \delta_{x, y} \delta_{s+1, s^{\prime}}+\left(1+\gamma_{5}\right) \delta_{x, y} \delta_{s-1, s^{\prime}}-2 \delta_{x, y} \delta_{s, s^{\prime}}\right] \\
+m\left[P_{R} \delta_{x, y} \delta_{s, 1} \delta_{s^{\prime}, L_{s}}+P_{L} \delta_{x, y} \delta_{s, L_{s}} \delta_{s^{\prime}, 1}\right]
\end{gathered}
$$

where $m$ is fermion mass, $P_{R, L}=\left(1 \pm \gamma_{5}\right) / 2, L_{s}$ the size of 5th dimension, and $D_{W}$ the standard Wilson kernel with negative mass.

Taking the limit of $L_{s} \rightarrow \infty$ with fixed $a_{s} L_{s}$, the action (마) accompanied by Pauli-Villars ghost term arrives at the overlap fermion operator that holds an exact chiral symmetry on the lattice expressed as the Ginsparg-Wilson relation.

In Ref. [四], we described our basic strategy based on the result of simulation with the SU(2) gauge theory with two flavors of fundamental fermions. In this paper, we extend the simulation to $N_{f}=2,4,6$, and 8 on a $16^{3} \times 32$ lattice.

\section{Setup of numerical simulation}

Numerical simulations were performed on a $16^{3} \times 32$ lattice with the Iwasaki gauge action and the standard domain-wall fermion action in the fundamental representation. For the latter, $L_{s}=16, M_{0}=1.6$ are adopted. To generate the gauge configurations, the hybrid Monte Carlo algorithm is employed with the Omelyan integrator and multi-time step acceleration of two levels. The simulation code is based on Bridge++ [W] with modification for the SU(2) gauge group and 


\begin{tabular}{ccccc}
\hline & & \multicolumn{3}{c}{$m$} \\
$N_{f}$ & $\beta$ & 0.20 & 0.10 & 0.05 \\
\hline 2 & 0.85 & $2.3419(77)$ & $2.623(14)$ & $2.827(22)$ \\
& 0.90 & $2.874(15)$ & $3.194(15)$ & $3.472(20)$ \\
\hline 4 & 0.85 & $2.842(21)$ & $3.551(43)$ & $4.215(89)$ \\
& 0.90 & $3.528(26)$ & $4.702(51)$ & $5.849(74)$ \\
\hline 6 & 0.80 & $2.462(13)$ & $3.403(32)$ & $4.851(83)$ \\
& 0.85 & $3.396(20)$ & $5.172(67)$ & $8.42(35)$ \\
& 0.90 & $4.611(46)$ & $6.551(90)$ & $10.61(52)$ \\
\hline 8 & 0.80 & $2.988(36)$ & $4.667(62)$ & $8.33(25)$ \\
& 0.85 & $4.317(44)$ & $7.15(16)$ & $15.9(1.1)$ \\
\hline
\end{tabular}

Table 1: Parameters of numerical simulations and the value of $r_{0}$ in each ensemble.
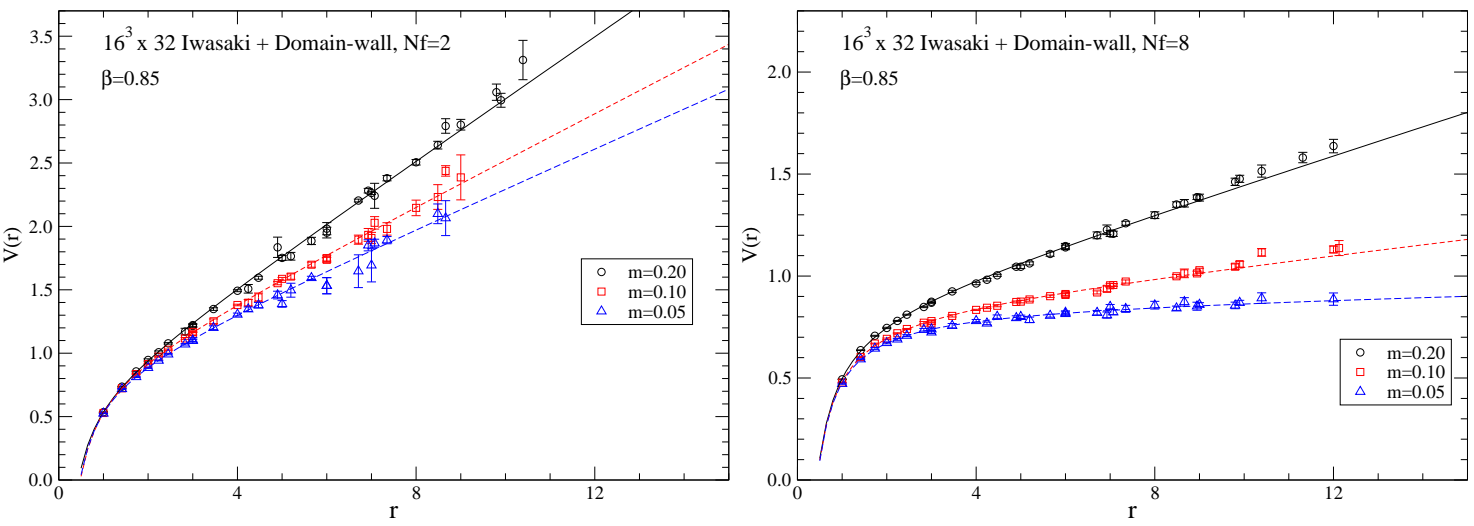

Figure 1: Static fermion potential on the $16^{3} \times 32$ lattice at $\beta=0.85$ for $N_{f}=2$ (left panel) and 8 (right).

the domain-wall fermions. So far, we have compiled at least 1,000 trajectories of unit length after 300 trajectories of thermalization. The parameter sets are listed in Table W.

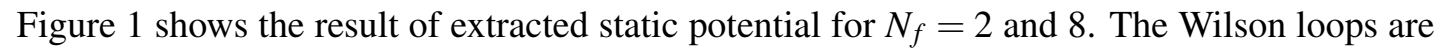
measured at every 5 trajectories with application of spatial link smearing. It is seen that the linear slope of the potential for $N_{f}=8$ is more sensitive to the fermion mass than $N_{f}=2$.

The potential is fitted to a standard form, $V(r)=C-A / r+\sigma r$. The hadronic radius $r_{0}$, which is defined through $\left[r^{2} \partial V(r) / \partial r\right]_{r_{0}}=1.65$, is determined from the result of the fit and listed in Table $\mathbb{W}$. While the SU(2) theory does not correspond to the real hadronic physics, just to display the lattice scale in a familiar manner, we determine the lattice spacing $a$ set by $r_{0}=0.49 \mathrm{fm}$. Figure $\square$ shows the result of $a\left(r_{0}\right)$ for $\beta=0.85$ and 0.90 against the bare fermion mass $m$ in lattice units. While the $m$ in the horizontal axis does not include the effect of the residual mass, the figure clearly shows that the fermion mass dependence becomes steeper as $N_{f}$ increases.

For $N_{f}=8$ an important question is whether the confining feature is lost or not in the chiral limit. Since the finite volume effect may become significant for smaller values of $m$, as indicated in large values of $r_{0}$, careful analysis is needed for conclusive result. 

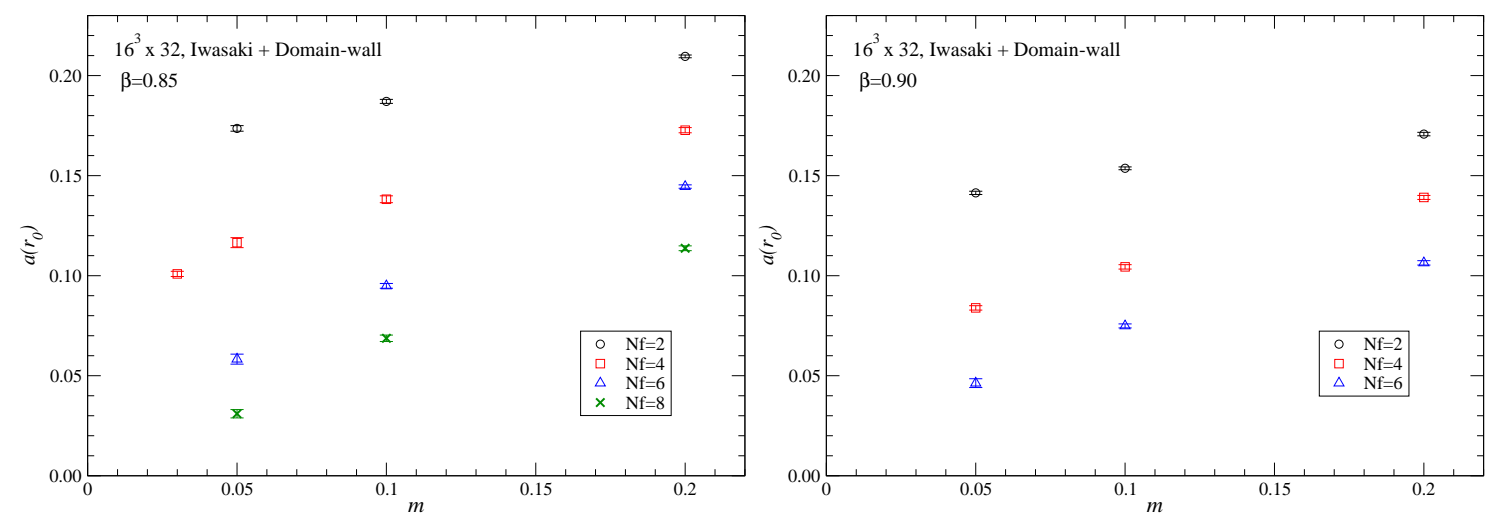

Figure 2: Lattice spacing $a\left(r_{0}\right)$ versus the bare fermion mass.

\section{Residual mass and meson masses}

For the meson spectrum, we note that the numerical cost is reduced thanks to the nature of the $\mathrm{SU}(2)$ gauge group. As proved in Ref. [目], the Wilson fermion operator $D_{W}$ for $\mathrm{SU}(2)$ gauge group has the symmetry

$$
D_{W}(x, y)=C^{-1}\left(-i \sigma_{2}\right)^{-1}\left[D_{W}(y, x)\right]^{T}\left(-i \sigma_{2}\right) C,
$$

and thus the corresponding relation for the propagator represents that the baryon and meson correlators are identical. Using $\gamma_{5}$-hermiticity, Eq. (B.J) implies for the propagator $S_{W}(x, y)=D_{W}^{-1}(x, y)$ that

$$
S_{W}(x, y)=C^{-1}\left(-i \sigma_{2}\right)^{-1} \gamma_{5} S_{W}(x, y)^{*} \gamma_{5}\left(-i \sigma_{2}\right) C,
$$

and thus the solutions of the linear equation for the source vectors of the second color component are determined from that of the first one. Similar relations also hold for the domain-wall fermion operator,

$$
\begin{aligned}
D_{D W}\left(x, s ; y, s^{\prime}\right) & =C^{-1}\left(-i \sigma_{2}\right)^{-1} R D_{D W}\left(y, s^{\prime} ; x, s\right)^{T} R C\left(-i \sigma_{2}\right), \\
& =C^{-1}\left(-i \sigma_{2}\right)^{-1} \gamma_{5} D_{D W}\left(x, s ; y, s^{\prime}\right)^{*} \gamma_{5} C\left(-i \sigma_{2}\right),
\end{aligned}
$$

where $R_{s, s^{\prime}}=\delta_{L_{s}-s+1, s^{\prime}}$ and the relation $D_{D W}^{\dagger}=\gamma_{5} R D_{D W} R \gamma_{5}$ was used. Thus a similar relation as Eq. (B.2) holds for the propagator of the domain-wall fermion. This reduces numerical cost for the linear equation solver.

In the domain-wall formulation, the residual mass $m_{\text {res }}$ serves as a probe to the explicit chiral symmetry breaking. It is defined through the overlap of the left and right handed modes at the center of the fifth direction, as

$$
R(t)=\frac{\sum_{\vec{x}}\left\langle J_{5 q}(\vec{x}, t) P(0)\right\rangle}{\sum_{\vec{x}}\langle P(\vec{x}, t) P(0)\rangle},
$$

where

$$
J_{5 q}(x)=-\bar{\psi}\left(x, L_{S} / 2\right) P_{L} \psi\left(x, L_{s} / 2+1\right)+\bar{\psi}\left(x, L_{s} / 2+1\right) P_{R} \psi\left(x, L_{s} / 2\right) .
$$

An average of $R(t)$ over large $t$ separation is a standard definition of the residual mass. Figure B] shows the residual mass for the valence fermion mass equal to the dynamical one, $m_{v a l}=m_{\text {sea }}$, against the lattice spacing. As a general tendency, as lattice spacing decreases, the residual mass 


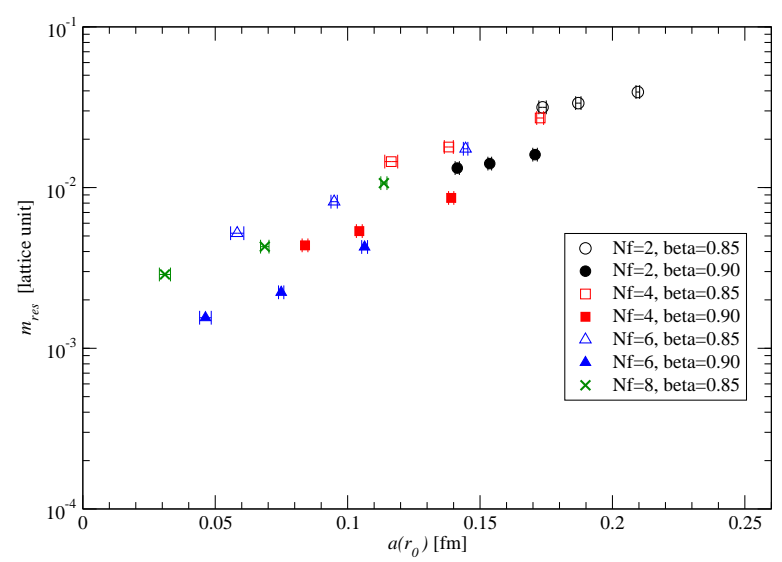

Figure 3: Residual mass in lattice units versus the lattice spacing $a\left(r_{0}\right)$.
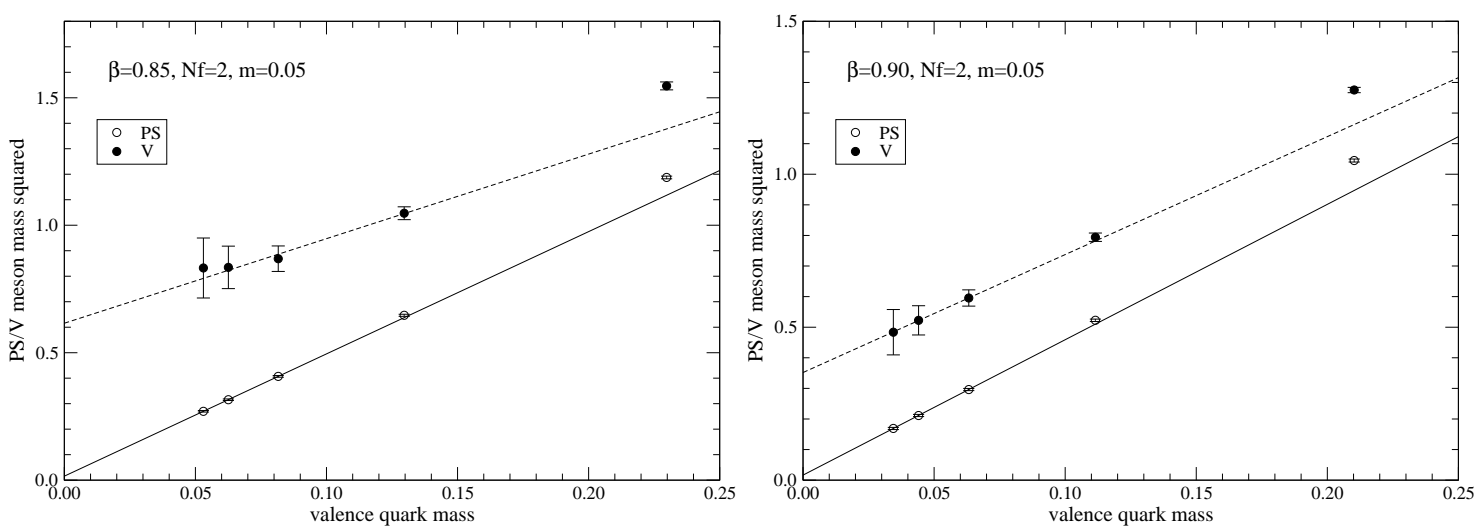

Figure 4: PS and V meson masses versus the valence fermion mass including the residual mass for $N_{f}=2$ at $(\beta, m)=(0.85,0.05)$ and $(0.90,0.05)$. The lines represent the results of linear fits using the smallest three points except for the case of the $\mathrm{V}$ meson at $\beta=0.85$ for which four points used.

decreases for fixed extent in the fifth direction $L_{s}$. Besides that, there is no significant $N_{f}$ dependence compared to the $\beta$ dependence. The residual mass is not negligibly small compared to the bare mass and causes considerable shift in fermion mass, in particular for the small $N_{f}$ cases in this work. To perform a simulation in the vicinity of the chiral limit, some improvement to reduce the residual mass is needed.

We measure the meson correlators in the pseudoscalar (PS), vector (V), connected part of scalar (S), and axialvector (A) channels with local meson operators in every 10 trajectories. As noted above, the baryon (diquark) correlators are the identical to the meson ones. Since for large $N_{f}$ the lattice spacing rapidly changes as fermion mass $m$ is varied, and numbers of values of $m$ is limited, we plot the meson masses as functions of the valence fermion mass $m_{v a l}$ for each ensemble.

Figure $⿴$ displays the present results for $N_{f}=2$. At $m=0.05$, we generate 2,000 trajectories of gauge configurations. As shown in the figure, the PS and V meson masses show similar behavior as in QCD. The valence fermion mass in the horizontal axis is now including the residual mass determined at each $m_{\text {val }}$. The $\mathrm{S}$ and A correlators suffer from large statistical fluctuation as seen in Fig. [1. It seems feasible to measure the masses and decay constants in A channel, while necessary to increase the statistics and to apply techniques to improve the signal. 

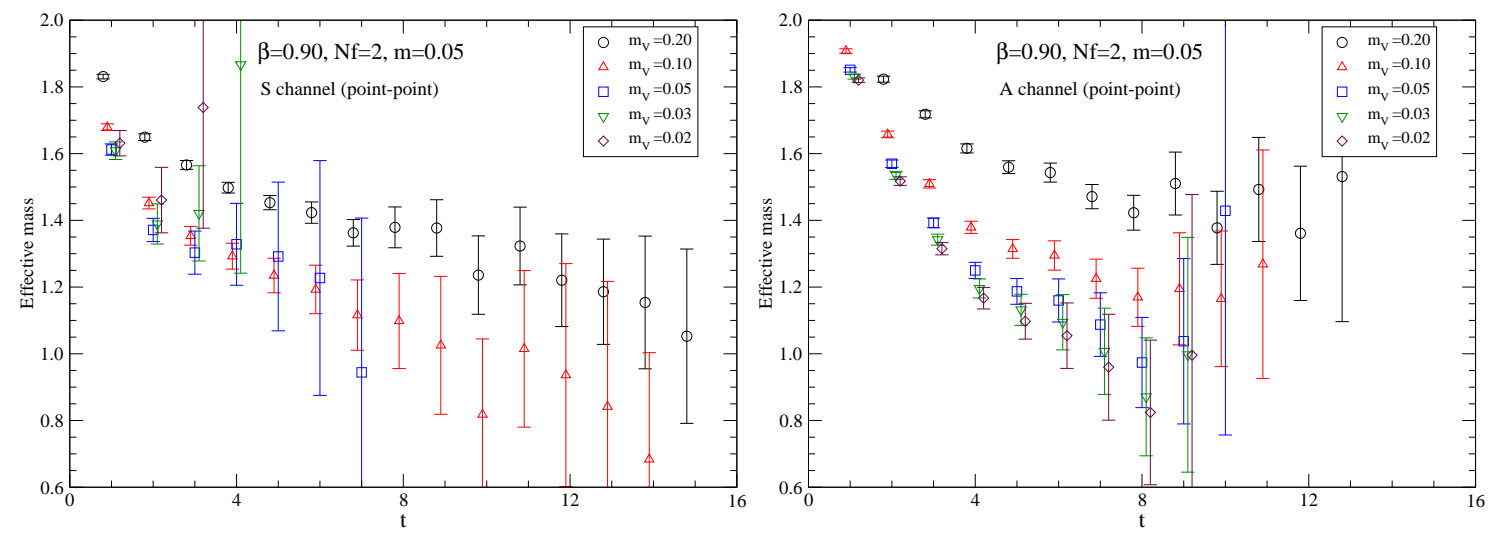

Figure 5: Effective mass plots for the $\mathrm{S}$ and A meson correlators for $N_{f}=2$ at $(\beta, m)=(0.90,0.05)$.
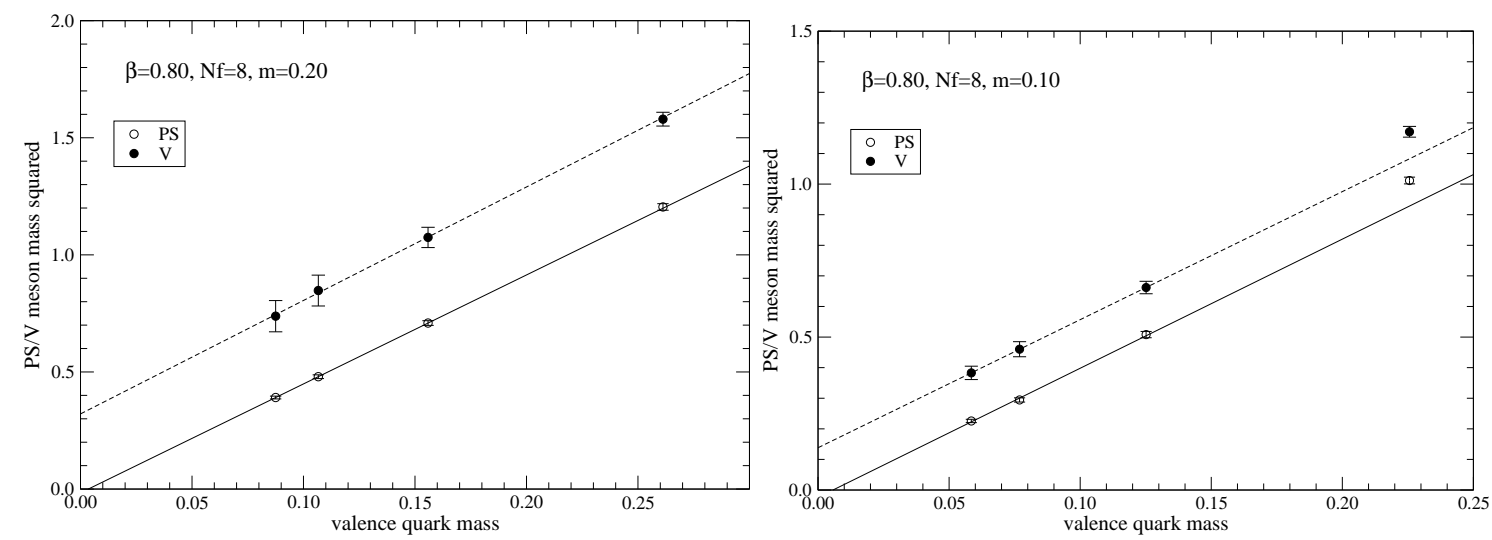

Figure 6: PS and V meson masses versus the valence fermion mass including the residual mass for $N_{f}=8$ at $(\beta, m)=(0.80,0.20)$ and $(0.80,0.10)$.

For $N_{f}$ other than 2, the meson spectra are measured in the same manner. On the ensembles, the PS and V meson masses show the similar behavior to the $N_{f}=2$ case. In particular, the PS meson masses obey the GMOR relation as a signal of spontaneous chiral symmetry breaking. Figure 6 shows the results for $N_{f}=8$. At $m=0.05$, the effective mass plots do not exhibit clear plateau when $m_{v a l}<0.05$. To extract the ground state signal, application of the smearing technique is now underway.

\section{Summary and outlook}

The SU(2) gauge theory is interesting because it has several unique properties distinct from SU(3) and hence its nonperturbative dynamics are not understood as well as SU(3). Motivated by this, we are performing numerical simulations of the $\mathrm{SU}(2)$ gauge theory with many flavors of chiral fermions, where simulations with smaller $N_{f}$ are aiming at shedding light on chiral symmetry breaking while large $N_{f}$ simulations are dedicated to the investigation of conformal dynamics. Qualitative difference has been already seen in the static potential or the mass dependence of the $m_{P} / m_{V}$ ratio between small and large $N_{f}$ theories. 
Once exploratory studies has been completed, there are many interesting extensions. It is interesting to study properties at finite temperature, especially the order of the phase transition. As for the nature of chiral symmetry breaking, the theory with adjoint fermions is attractive since it has another different breaking pattern, $S U\left(2 N_{f}\right) \rightarrow S O\left(2 N_{f}\right)$. Our attempt in the adjoint representation was presented in [12]. The study of nonperturbative aspects of the Glashow-Weinberg-Salam model is also one of the possible directions [[13]. Thus for future studies, accumulating fundamental data is clearly important, which is another task to be done in this work.

\section{Acknowledgment}

Numerical simulations were performed on Hitachi SR16000 and IBM Blue Gene/Q at KEK under a support of its Large-scale Simulation Program (Nos.12/13-15, 13/14-14) and $\varphi$ computer system at KMI, Nagoya University. We also thank the Japan Lattice Data Grid which is a grid file system constructed on a virtual private network SINET4 provided by National Institute of Informatics for efficient data transfer. This work is supported in part by the Grand-in-Aid for Scientific Research of Japan (Nos.22224003, 22740183, 25400284).

\section{References}

[1] K. Yamawaki, M. Bando and K. i. Matumoto, Phys. Rev. Lett. 56, 1335 (1986).

[2] For reviews on technicolor model, see, for example, C. T. Hill and E. H. Simmons, Phys. Rept. 381, 235 (2003) [Erratum-ibid. 390, 553 (2004)] [hep-ph/0203079]; F. Sannino, arXiv:0804.0182 [hep-ph].

[3] For recent activities on the lattice, see, for example, J. Kuti, PoS LATTICE 2013, 004 (2014).

[4] F. Bursa, L. Del Debbio, L. Keegan, C. Pica and T. Pickup, Phys. Lett. B 696, 374 (2011) [arXiv:1007.3067 [hep-ph]].

[5] H. Ohki, T. Aoyama, E. Itou, M. Kurachi, C.-J. D. Lin, H. Matsufuru, T. Onogi, E. Shintani, and T. Yamazaki, PoS LATTICE 2010, 066 (2010) [arXiv:1011.0373 [hep-lat]].

[6] R. Lewis, C. Pica and F. Sannino, Phys. Rev. D 85, 014504 (2012) [arXiv:1109.3513 [hep-ph]].

[7] T. Karavirta, J. Rantaharju, K. Rummukainen and K. Tuominen, JHEP 1205, 003 (2012) [arXiv:1111.4104 [hep-lat]].

[8] M. Hayakawa, K.-I. Ishikawa, S. Takeda, M. Tomii and N. Yamada, Phys. Rev. D 88, 094506 (2013) [arXiv:1307.6696 [hep-lat]].

[9] M. Hayakawa, K.-I. Ishikawa, S. Takeda and N. Yamada, Phys. Rev. D 88, 094504 (2013) [arXiv:1307.6997 [hep-lat]].

[10] H. Matsufuru, Y. Kikukawa, K. i. Nagai and N. Yamada, PoS LATTICE 2013, 123 (2014) [arXiv:1401.6655 [hep-lat]].

[11] S. Ueda et al., PoS LATTICE 2013, 412 (2014); J. Phys. Conf. Ser. 523, 012046 (2014).

[12] H. Matsufuru, Y. Kikukawa, K. -I. Nagai and N. Yamada, PoS LATTICE 2010, 090 (2010); H. Matsufuru, Y. Kikukawa and N. Yamada, PoS LAT 2009, 064 (2009).

[13] D. Kadoh and Y. Kikukawa, JHEP 0805, 095 (2008) [Erratum-ibid. 1103, 095 (2011)] [arXiv:0709.3658 [hep-lat]]. 